\title{
Extended liver resection including hypertrophy concept with portal venous embolisation for giant haemangioma. Too much surgery?
}

\author{
Alina Strohmaier ${ }^{1,2}$, Kim C. Wagner ${ }^{1,2}$, Tim Reese ${ }^{1,2}$, Mohammad Fard-Aghaie ${ }^{1,2}$, \\ Georgios Makridis $^{1,2}$, York von Rittberg ${ }^{1,2}$, Katja Horling ${ }^{3}$, and Karl J. Oldhafer ${ }^{1,2}$ \\ ${ }^{1}$ Division of Hepatobiliary and Pancreatic Surgery, Department of Surgery, Asklepios Hospital Barmbek, \\ ${ }^{2}$ Semmelweis University of Medicine, Asklepios Campus Hamburg, \\ ${ }^{3} \mathrm{MVZ}$ Hanse Histologikum GmbH, Hamburg, Germany
}

\begin{abstract}
Haemangiomas of the liver are benign tumours, which are often diagnosed randomly. With an increase in size haemangiomas could become symptomatic. In this case therapeutic options, surgical or interventional, have to be weighted to a conservative approach. We present a case of a 36-year old woman with a symptomatic giant haemangioma of the right liver lobe. Because of the size of the tumor and the small future liver remnant we decided to perform a major liver resection after hypertrophy induction with a preoperative portal vein embolization; an option mainly used for major hepatectomies in malignant tumors of the liver. But however, this case shows, that using a hypertrophy concept also for benign liver tumours is the safer approach, if an extended resection is necessary and the future liver remnant is critical. (Ann Hepatobiliary Pancreat Surg 2020;24:357-361)
\end{abstract}

Key Words: Haemangioma; Liver resection; Portal venous embolization

\section{INTRODUCTION}

As the most common type of benign mesenchymal liver tumors, haemangiomas are often asymptomatic and diagnosed incidentally. ${ }^{1}$ In general, a conservative therapy approach with imaging follow-up, using ultrasound or MRI scan at regular intervals can be recommended. Due to its benign course, imaging follow-up is not even required for typical haemangiomas. The necessity for treatment of hemangiomas can be discussed by the presence of symptoms. ${ }^{2}$ When treatment is indicated, surgery is therapy of choice and enucleation of the haemangioma should be performed. As a non-surgical approach a transarterial chemoembolization (TACE) can be performed to reduce the tumor growth. ${ }^{3}$ In our case the haemangioma was giant and symptomatic, therefore surgical therapy was indicated, which was, however, challenging due to size and location.

\section{CASE}

A 36-year old female was diagnosed with a size progressive giant haemangioma of the right liver lobe. Initially it was diagnosed in 2008 during a routine ultrasound. It measured $6.5 \mathrm{~cm}$ and was asymptomatic. In January 2019 the patient started to develop abdominal pain of the right upper quadrant, bloating, nausea and vomiting, which led to a weight loss of $10 \mathrm{~kg}$ within 6 months. On physical examination the liver was greatly enlarged with a palpable mass into the pelvic region (Fig. 1). No ascites, signs of jaundice or lower extremity edema where observed. She had no history of viral hepatitis, had taken oral contraceptives in early years and carried a pregnancy 11 years ago. An abdominal computed tomography (CT) scan revealed a $16 \times 22 \times 23 \mathrm{~cm}$ haemangioma filling the right liver lobe and segment 1 almost completely (Fig. 2). Therefore the only treatment option was the resection. A safe enucleation of the haemangioma with preservation

Received: February 8, 2020; Revised: April 19, 2020; Accepted: April 29, 2020

Corresponding author: Tim Reese

Division of Hepatobiliary and Pancreatic Surgery, Department of Surgery, Asklepios Hospital Barmbek, Rübenkamp 220, Hamburg 22307, Germany

Tel: +49-40-1818-82-8114, Fax: +49-40-1818-82-2819, E-mail: t.reese@asklepios.com

Copyright (C) 2020 by The Korean Association of Hepato-Biliary-Pancreatic Surgery

This is an Open Access article distributed under the terms of the Creative Commons Attribution Non-Commercial License (http://creativecommons.org/ icenses/by-nc/4.0) which permits unrestricted non-commercial use, distribution, and reproduction in any medium, provided the original work is properly cited. Annals of Hepato-Biliary-Pancreatic Surgery • pISSN: 2508-5778 - eISSN: 2508-5859 

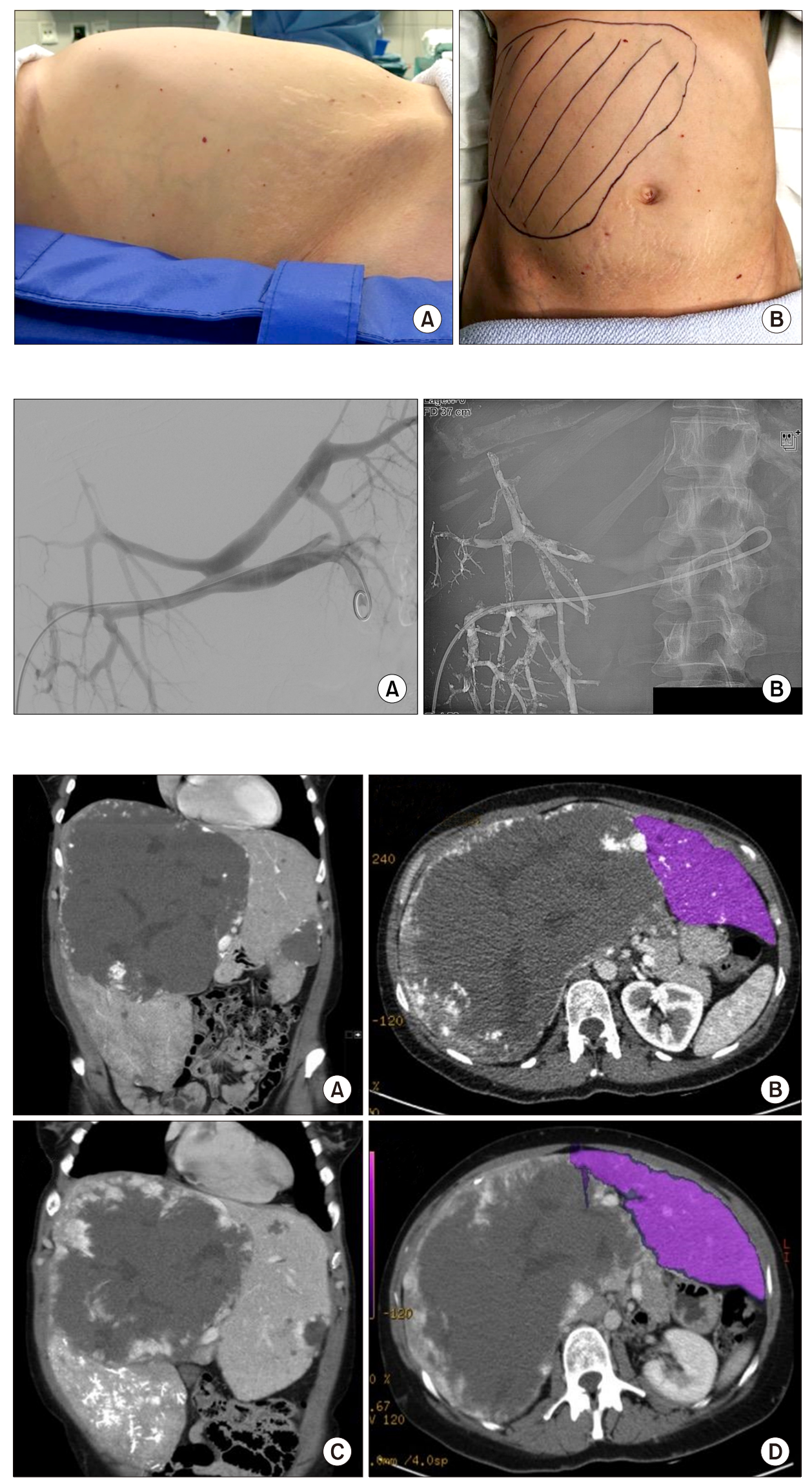

Fig. 1. Clinical findings. (A) Photograph from the right patient side. Distended abdomen due to enlarged liver. (B) Marking of the palpabel liver.

Fig. 2. Portal Vein Embolisation of all right hepatic portal veins. (A) Angiography of the portal vein with a five FrenchPigtail-Catheter. The catheter is placed in the main portal vein. (B) Portal vein embolization with Glubran of the right anterior and posterior branch.
Fig. 3. CT-Scans. (A) Giant haemangioma in the right lobe of the liver and smaller haemangioma in segment III. (B) Volumetric CT scan of the left liver lobe. (C) CT Scan 2 weeks after PVE showing an enlarged left liver lobe. (D) Volumetric CT scan of the left liver lobe after PVE. 
of healthy liver parenchyma in the right lobe seemed only possible when complete vascular isolation of the liver with hypothermic perfusion was used. The surgical alternative to this high risk procedure was a right trisectorectomy including segment I. But due to the small volume of segments II and III, there would be a high risk of postoperative liver failure. To reduce that risk we chose a hypertrophy concept and the patient received a portal venous embolisation (PVE) of all right portal vein branches to induce the growth of the future liver remnant (FLR) (Fig. 2). After PVE the volume of the FLR increased from 873 $\mathrm{ml}$ to $1.030 \mathrm{ml}$ (sFLR of $69.9 \%$; BSA (Mosteller): 1.79 $\mathrm{m}^{2}$; TELV (Vauthey): $1473 \mathrm{~cm}^{3}$ ) within 2 weeks (Fig. 3B,
C). Surgery was planned 4 weeks later. Intraoperatively a huge right liver lobe was found, reaching into the pelvis (Fig. 4A-C). The entire liver hilum was stretched over the enlarged liver segment I (Fig. 4D). The left liver lobe was also clearly enlarged. Intraoperative ultrasound showed at least two additional small haemangiomas in the left liver lobe. After dissecting the right hepatic artery, the right portal vein branches, the right bile duct and the right and middle hepatic veins liver segments IV-VIII and segment I were removed en bloc. A T-drainage was inserted into the common hepatic bile duct. The inferior vena cava was clamped for $45 \mathrm{~min}$, because of bleeding complications from the hepatic veins. Pringle maneuver was applied for
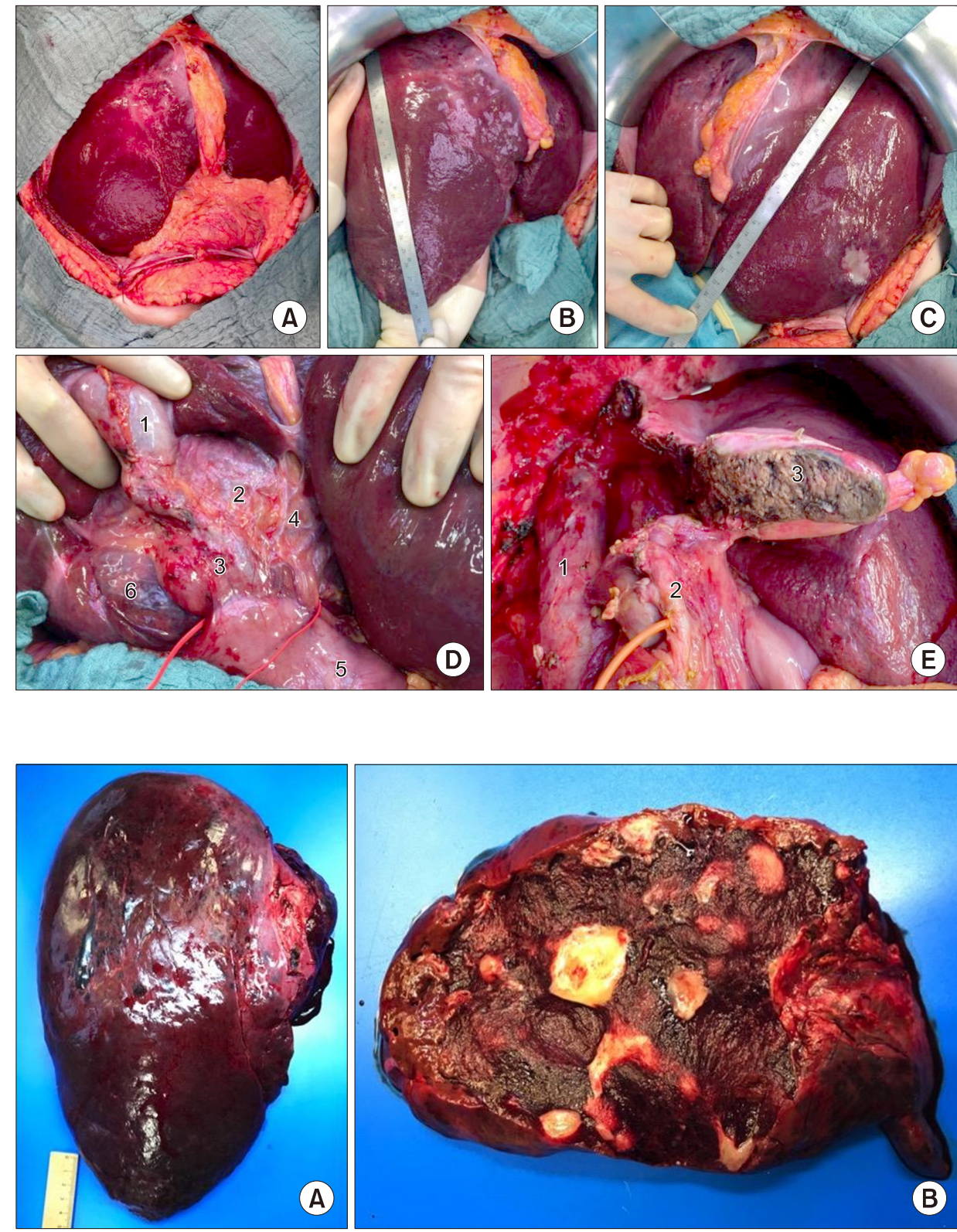

Fig. 5. Pathological findings. (A) Specimen of the extended right resection (liver segment IV-VIII and I); (B) Cross section with characteristic structure of haemangioma with small streak of regular liver parenchyma on the left margin. 

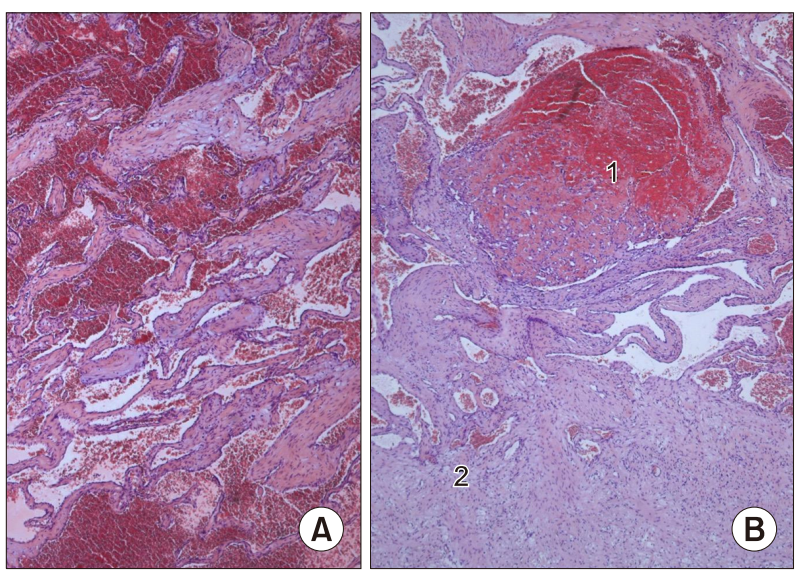

Fig. 6. (A) HE-stained sections showed the classic morphology of a cavernous haemangioma with widely dilated vascular channels lined by flattened inconspicuous endothelial cells and fibrous walls, (B) focal organized thrombi (1) and partial sclerotic areas (2) could be recognised.

$10 \mathrm{~min}$. The intraoperative blood loss was $1100 \mathrm{ml}$. The ICU-stay was 3 days. The postoperative course was uneventful. There was no sign of a bile-leakage, so the T-drain was removed early. The patient was discharged from the hospital after 12 days without any further complications or complaints.

The gross processing showed a liver specimen of the $2,100 \mathrm{~g}$ weight with a solitary red-brown and well-demarcated $17 \mathrm{~cm}$ tumour with spongy cut surface and some fibrotic areas (Fig. 5). Histological examination showed a haemangioma with a predominantly cavernous architecture (most common subtype) with focal thrombi and regressive fibrosis (Fig. 6). The adjacent liver tissue presented an overall preserved architecture with peliosis hepatis like change and low to moderate portal field fibrosis, lymphocytic portal field infiltrates and minimal cholestasis (Fig. 7).

\section{DISCUSSION}

In general haemangiomas of the liver are small $(<1$ $\mathrm{cm}$ ), asymptomatic and do not require any treatment. Most commonly monitoring via imaging techniques is recommended. But however, so called giant haemangiomas are different. They are defined as greater than $5 \mathrm{~cm}$, hypergiant haemangiomas as $>10 \mathrm{~cm}^{4}$ Due to their size they can cause various complaints and often need some form of treatment. Transarterial chemoembolization (TACE),

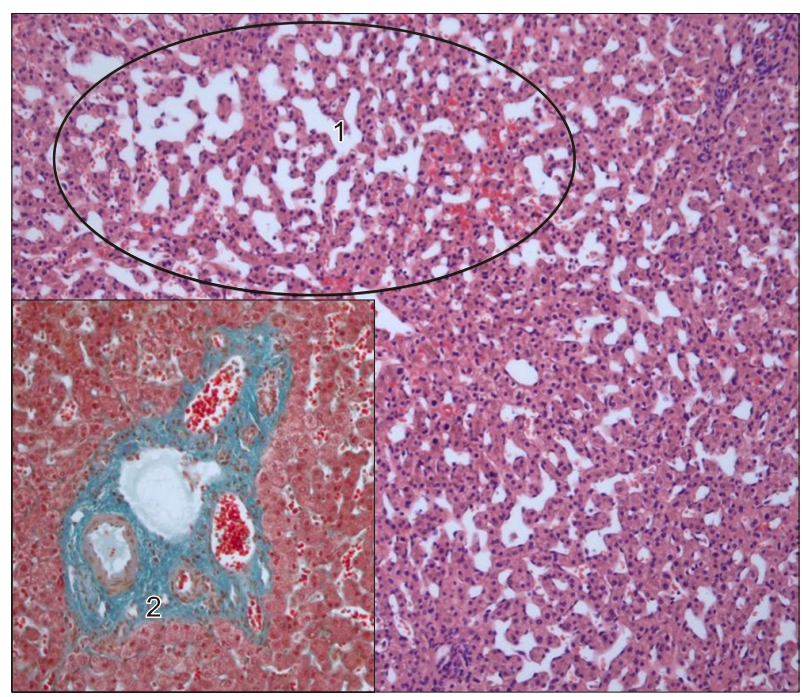

Fig. 7. Adjacent liver parenchyma with peliosis hepatis like sinusoidal dilatation (1) and mild fibrosis of the portal tracts in Elastica van Gieson stain (2).

radiofrequency (RFA) or microwave ablations (MWA) are possible non-surgical treatment strategies for haemangiomas, which can be applied to decrease the growth. Nevertheless, these treatments are reserved for minor haemangiomas. ${ }^{5-7}$ Although non-operative management might be the right approach for the majority of patients, patients with abdominal compression symptoms are more likely to benefit from surgery, as it is the most effective therapy. ${ }^{8}$ In our case the patient was not likely to benefit from any non-surgical treatment. The complaints led to an immense loss of quality of life, as food-intake was almost not possible. Therefore, different surgical strategies needed to be discussed. A conventional right hemihepatectomy (segment V-VIII) would not have been sufficient, because the haemangioma advanced into liver segments IVa and b. An enucleation of the tumour would have been due to the high risk of major complication, severe blood loss for example, and only marginal healthy liver parenchyma not favourable either. Hence, we decided to perform a right trisectorectomy, using a preoperative PVE to induce hypertrophy of the remaining segments II and III. PVE is a valuable tool to successfully induce hypertrophy of the future liver remnant prior to surgical resection. In hepatic surgery hypertrophy concepts before resection are almost exclusively applied to malignant tumours of the liver. PVE was initially used in order to increase the resectability rates in patients with cholangiocarcinoma (CCC) 
and hepatocellular carcinoma (HCC) to decrease postoperative mortality. However over the last twenty years indications for PVE have been expanded and also include liver metastasis and other tumours with small FLR prior to major liver resection. ${ }^{9}$ In our case major hepatectomy without PVE would have increased the risk of post hepatectomy liver failure. To our knowledge, this case describes the first major hepatectomy following PVE in a patient with a hypergiant haemangioma in the literature. Previously described surgical treatments were TACE before surgical resection, enucleation or conventional right or left liver resection. ${ }^{4,10}$

\section{ORCID}

Alina Strohmaier: https://orcid.org/0000-0001-5260-6037

Kim C. Wagner: https://orcid.org/0000-0001-7109-1833

Tim Reese: https://orcid.org/0000-0002-9163-5962

Mohammad Fard-Aghaie:

https://orcid.org/0000-0002-5798-6590

Georgios Makridis: https://orcid.org/0000-0001-6187-9336

York von Rittberg: https://orcid.org/0000-0001-6804-274X

Katja Horling: https://orcid.org/0000-0003-4939-7312

Karl J. Oldhafer: https://orcid.org/0000-0002-4878-265X

\section{REFERENCES}

1. Farges O, Daradkeh S, Bismuth H. Cavernous hemangiomas of the liver: are there any indications for resection? World J Surg 1995; 19:19-24.

2. Fodor M, Primavesi F, Braunwarth E, Cardini B, Resch T, Bale $\mathrm{R}$, et al. Indications for liver surgery in benign tumours. Eur Surg 2018;50:125-131.

3. Furumaya A, van Rosmalen BV, Takkenberg RB, van Delden OM, Dejong CHC, Verheij J, et al. Transarterial (chemo-)embolization and lipiodolization for hepatic haemangioma. Cardiovasc Intervent Radiol 2019;42:800-811.

4. Adhikari DR, Thakur V, Telavane PP, Kulkarni R, Singh R, Joshi RM. Hypergiant hepatic hemangiomas: case series. Indian J Surg 2015;77(Suppl 1):40-42.

5. Firouznia K, Ghanaati H, Alavian SM, Nassiri Toosi M, Ebrahimi Daryani N, Jalali AH, et al. Management of liver hemangioma using trans-catheter arterial embolization. Hepat Mon 2014; 14:e25788.

6. Gao J, Ke S, Ding XM, Zhou YM, Qian XJ, Sun WB. Radiofrequency ablation for large hepatic hemangiomas: initial experience and lessons. Surgery 2013;153:78-85.

7. Ziemlewicz TJ, Wells SA, Lubner MA, Musat AI, Hinshaw JL, Cohn AR, et al. Microwave ablation of giant hepatic cavernous hemangiomas. Cardiovasc Intervent Radiol 2014;37:1299-1305.

8. Duxbury MS, Garden OJ. Giant haemangioma of the liver: observation or resection? Dig Surg 2010;27:7-11.

9. Glantzounis GK, Tokidis E, Basourakos SP, Ntzani EE, Lianos GD, Pentheroudakis G. The role of portal vein embolization in the surgical management of primary hepatobiliary cancers. A systematic review. Eur J Surg Oncol 2017;43:32-41.

10. Ketchum WA, Lin-Hurtubise KM, Ochmanek E, Ishihara K, Rice RD. Management of symptomatic hepatic "mega" hemangioma. Hawaii J Med Public Health 2019;78:128-131. 